\title{
Network Optimization through Simulation Model
}

\author{
Dr. Santosh Deshpande \\ Professor (H.O.D), MES’S IMCC (M.C.A Department) \\ Savitribai Phule Pune University
}

\begin{abstract}
The paper presents the results of a detailed packet-level simulation comparing three multi-hop wireless ad hoc network routing protocols under the load of different probability distributions, that cover a range of design choices having different protocol viz. AODV,OLSR and TORA. We have extended the OPNET network simulator to accurately model the MAC and physical-layer behavior of the IEEE 802.11 wireless LAN standard, including a realistic wireless transmission channel model. Simulation of $\mathbf{1 0 0}$ mobile nodes has been carried out and the performance optimization is determined.
\end{abstract}

Keywords - Simulation, Opnet, Wireless, Statistical probability distribution, IEEE802.11, throughput, delay, retransmission attempt, load, protocol, MAC, LAN

\section{INTRODUCTION}

Ad-hoc wireless network is that network where no communication is present, in such network; each mobile node operates not only as a host but also as router. Mobile nodes in the network may not be within range of each other, communication of these nodes perform by discovering "multi-hop" paths through the network to other nodes. This type of network is some time called infrastructure less network [1]. Some examples of the possible uses of ad hoc networking are students using laptop computers to participate in an interactive lecture, business associates sharing information during a meeting, soldiers relaying information for situational awareness on the battlefield [2,3]. Many different protocols have been proposed to solve the multi hop routing problem in ad hoc networks, each based on different assumptions and intuitions.

Mobile Ad hoc Networks (MANETs)[1] are an emerging technology that allows establishing an instant communication network for civilian and military applications, without relying on pre-existing fixed network infrastructure. The nodes in a MANET can dynamically join and leave the network, frequently, often without warming, and possibly without disruption to other nodes' communication. Each node in the network also acts as router, forwarding data packet for other nodes. A central challenge in design of Ad hoc network is the development of dynamic routing protocols that can effectively find the route between two communicating nodes. The routing protocol must be able to keep up with the high degree of node mobility that often changes the topology drastically and unpredictably.

The current Mobile Ad Hoc Network (MANET) [2] paradigm as described by the Internet Engineering Task Force (IETF) MANET work group. Routing algorithms are often difficult to formalize into mathematics; they are instead tested using extensive simulation. A large amount of work has been done in the area of energy efficient routing. This approach attempts to maximize network lifetime by routing through paths, which use the least amount of energy relative to each node. Now a day, more attention has been given to use specific network parameters while specifying routing matrixes. Routing matrixes includes delay of network, link capacity, link stability or identifying low mobility nodes. These schemes are generally based on previous work, which is then enhanced with the new matrix.

The paper is providing a realistic, quantitative analysis comparing the performance of a variety of multi-hop wireless ad hoc network routing protocols. We present results of detailed simulations showing the relative performance of three recently proposed ad hoc routing protocols: AODV [4], OLSR [6] and TORA [7].

Our results in this paper are based on simulations of an ad hoc network of 100 wireless mobile nodes moving about and communicating with each other. We analyze the performance of each protocol and explain the design choices that account for their performance.

The section 2 of the paper describes the different types of protocols used in the simulation. The section 3 has given description of design of simulation model. The performance analysis is describes in section 4 and the section 5 has summaries with conclusion of the paper. 


\section{DESCRIPTION OF PROTOCOLS}

\subsection{Ad Hoc on demand Vector (AODV) [4]}

AODV discovers routes on demand basis. It uses routing table to maintain routing information, one entry per destination. RREP packet is used to replies back to the source and, subsequently, to route data packets to the destination. AODV uses sequence numbers to maintain at each destination to determine routing information and to prevent routing loops [4]. AODV working on timer- based states in each node. A routing table entry is expired if not used recently. If node link is broken, the all predecessor nodes forward the RERR packets, to effectively erasing all routes using broken link. AODV uses expanding ring search technique initially to discover routes to an unknown destination. AODV algorithm has the ability to quickly adapt to dynamic link conditions with low processing and memory overhead. AODV offers low network utilization and uses destination sequence number to ensure loop freedom AODV keeps the following information with each route table entry.

(i) Destination IP address (IP address for the destination node),

(ii) Destination sequence number,

(iii) Valid destination sequence number flag,

(iv) Network interface,

(v) Hop count, that is, number of hops required to reach the destination,

(vi) Next hop (the next valid node that did not re broadcast the RREQ message),

(vii) List of precursor,

(viii) Life time, that is, expiration or deletion time of a route.

\subsection{Optimized Link State Routing (OLSR) [6]}

The OLSR model implements the MPR (Multi Point Relay) flooding mechanism to broadcast and flood Topology Control (TC) messages in the network. The algorithm is implemented as suggested in OLSR RFC 3626. This mechanism takes advantage of controlled flooding by allowing only selected nodes (MPR nodes) to flood the TC message. Each node selects an MPR to reach its two-hop neighbors The OLSR model implements the neighbor sensing mechanism through periodic broadcast of Hello messages. These Hello messages are one-hop broadcasts (never forwarded) that carry neighbor type and neighbor quality information. The neighbor sensing mechanism provides information on up to two-hop neighbors. Generation and processing of the Hello messages are implemented as suggested in the OLSR RFC.

Periodic and triggered Topology Control (TC) messages implement the topology discovery/diffusion mechanism in the OLSR model. TC messages are generated by MPR nodes and carry information about MPR selector nodes. These messages are diffused throughout the network using controlled flooding, thus helping to form a topology of reachable nodes, previous hop on each node.

\subsection{Temporally Ordered Routing Algorithm (TORA) [7]}

The Temporally-Ordered Routing Algorithm (TORA) is an adaptive routing protocol for multi hop networks. It possesses the following attributes:

(i) Distributed execution,

(ii) Loop-free routing,

(iii) Multipath routing,

(iv) Reactive or proactive route establishment and maintenance

(v) Minimization of communication overhead via localization of algorithmic reaction to topological changes when possible. 
Its operation can be biased towards high reactivity (i.e. low time complexity) and bandwidth conservation (i.e. low communication complexity) rather than routing optimality (i.e. continuous shortest-path computation). Its design and flexibility make it potentially well-suited for use of mobile ad hoc networks (MANETs).

A key concept in the protocol's design is an attempt to de-couple (to the greatest extent possible) the generation of far-reaching control message propagation from the dynamics of the network topology. The scope of TORA's control messaging is typically localized to a very small set of nodes near a topological change. TORA includes a secondary mechanism that is independent of network topology dynamics. It allows far-reaching control message propagation as a means of route optimization or soft-state route verification

\section{MOBILE AD HOC NETWORK MODEL}

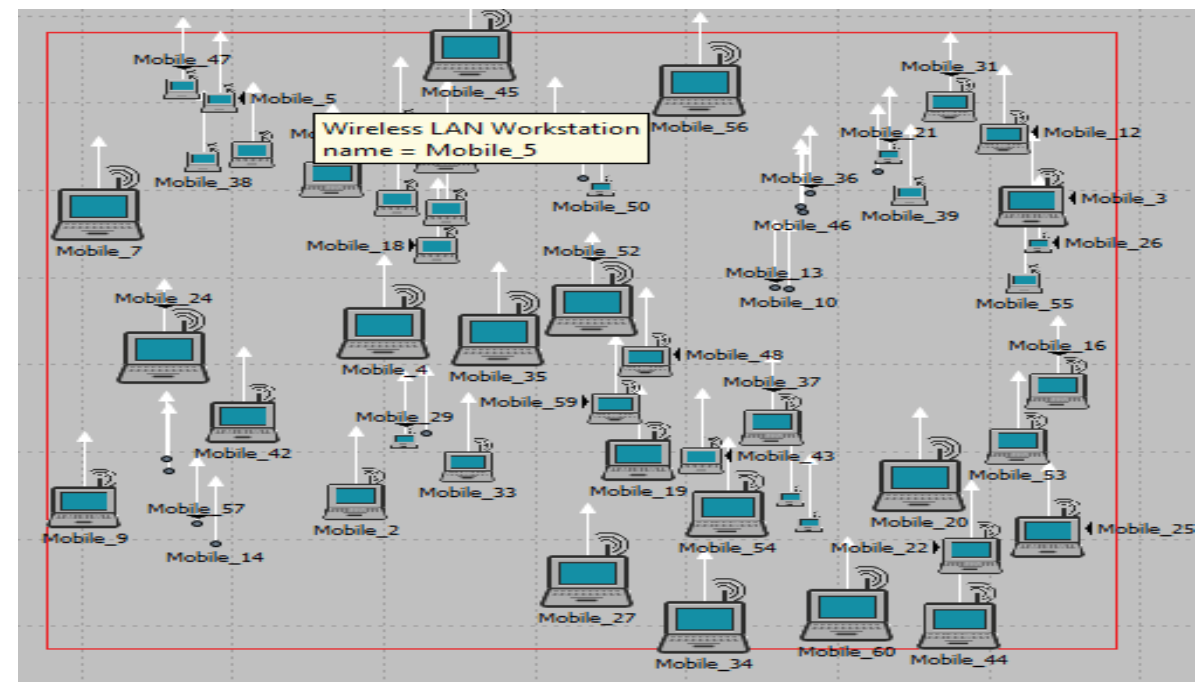

Figure 3.1 Ad hoc Wireless Network

We have design three Mobile Ad-hoc networks scenarios which consist of 100 mobile wireless nodes. Every scenario is following one routing protocol. Scenario one is based on AODV routing protocol, scenario two is following OLSR routing protocol and TORA routing protocol is used in scenario three. In order to enable direct, fair comparisons between Ad-hoc routing protocols, each protocol ran with identical load and environment conditions. Total simulation time was 3600 seconds with $128 \mathrm{kbps}$ speed.

As shown in the figure 3.1, wireless network consist of Wireless LAN workstations and supported by random mobility model. Wireless LAN workstation the "wlan_wkstn" model can be configured to run any MANET routing protocol. It is able to generate application traffic (FTP, E-mail, HTTP, etc.) and route the traffic using the MANET routing protocol configured.

\section{PERFORMANCE ANALYSIS OF PROTOCOL}

We have conducted relative performance study of Ad hoc routing protocols through simulation model using Optimize Network Engineering Tool (OPNET 14.5) simulator to carry out simulation. Performance of simulation has analysis based on following matrix.

a) Wireless LAN Throughput

b) End to End delay

c) Wireless LAN Data Dropped 


\section{4. (a) Wireless LAN Throughput}

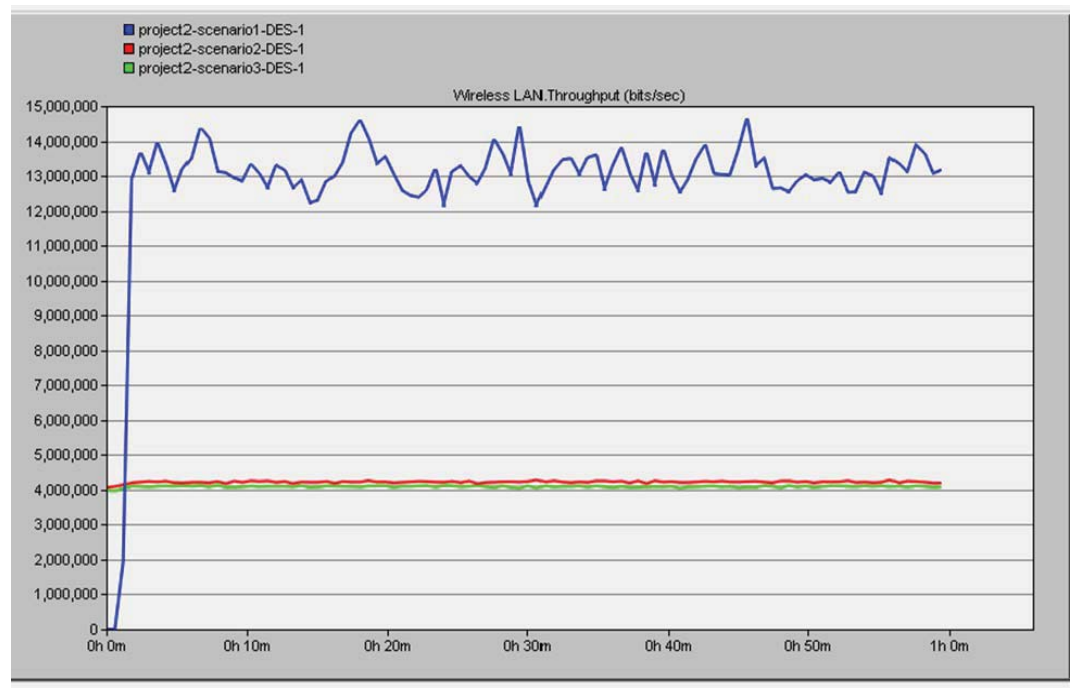

Figure 4.1 Wireless LAN Throughputs

As shown in the figure 4.1 throughput of Scenario one is far better than scenario two and three. Scenario one Mobile Ad hoc Network is based on AODV routing protocol and scenario two and three is based on OLSR and TORA routing protocol respectively. AODV is reactive protocol, its shows better throughput as compare to OLSR and TORA. OLSR and TORA are proactive protocols which shows stable throughput throughout the network simulation.

\section{4.(b) End to End delay}

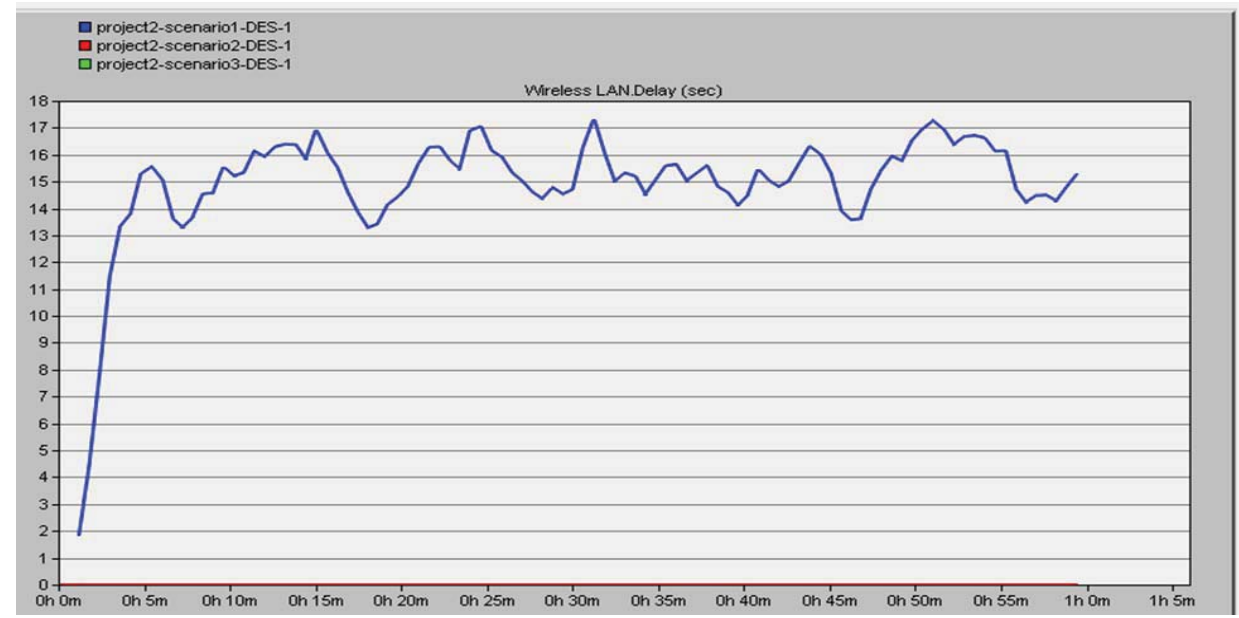

Figure 4.2 Wireless LAN Delay (Sec)

Scenario two and three shows no delay while transmitting the packets from one place to another as shown in the figure 4.2. These two scenarios are based on OLSR and TORA protocol. Scenario one shows fluctuating delay from 13 to $17 \mathrm{sec}$ throughout the simulation time. Scenario one is based on AODV protocol. AODV is dynamic in nature as well as reactive. AODV find the new path on every moment of the wireless mobile node. 


\section{4.(c) Wireless LAN Data Dropped (Buffer Overflow)}

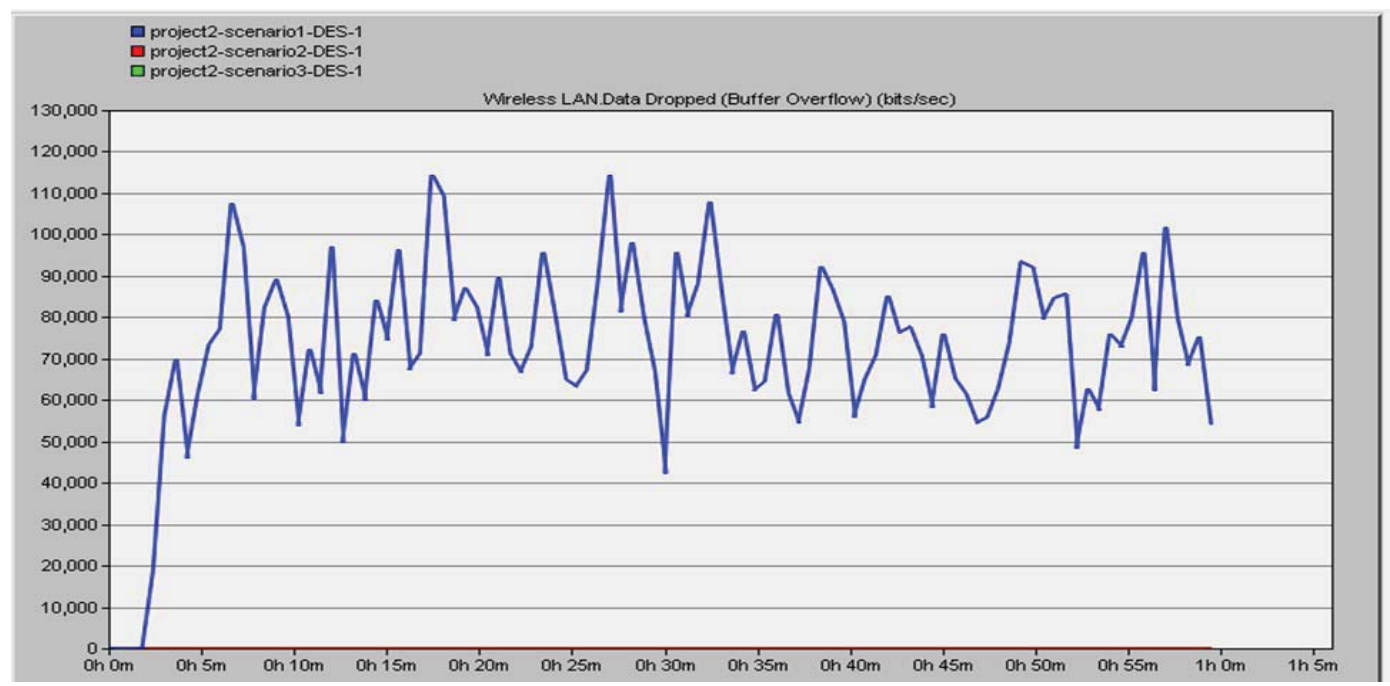

Figure 4.3 Wireless LAN Data Dropped (Bits/sec)

As shown in the figure 4.3 scenario one was shown data dropped due to the buffer overflow and scenario two and three shown no data dropped. On an average data dropped 70,000 bits/sec are reported after simulation of the model is completed. It was observed in the scenario one data dropped fluctuating from $45000 \mathrm{bits} / \mathrm{sec}$ to $115000 \mathrm{bits} / \mathrm{sec}$ throughout the simulation. When bits are reaching to the destination node, memory of the node was full and hence data was dropped by the node.

\section{SUMMARY AND CONCLUSIONS}

A MANET simulation models were developed for different Wireless routing protocols i.e. AODV, OLSR and TORA. The performance of simulation models was observed and discussed in above sections. Based on the performance of routing protocols following are the by and large observations.

1. The AODV performance is best in terms of throughput as compared to the OLSR and TORA.

2. OLSR and TORA have shown zero data dropped and zero delay,

3. It is observed that AODV has shown maximum delay and data dropped as compared to other routing protocols.

\section{REFERENCES}

[1] National Science Foundation. Research priorities in wireless and mobile communications and networking: Report of a workshop held March 24-26, 1997, Airlie House, Virginia. Available at http://www.cise.nsf.gov/anir/ww.html.E. Balaguruswamy, "engineering reliability" Tata mc. Graw Hill, New Delhi.

[2] Barry M. Leiner, Robert J. Ruth, and Ambatipudi R. Sastry. Goals and challenges of the DARPA GloMo program. IEEE Personal Communications, 3(6):34-43.

[3] David B. Johnson. Routing in ad hoc networks of mobile hosts. In Proceedings of the IEEE Workshop on Mobile Computing Systems and Applications, pages 158-163,

[4] C. Perkins, E. Belding-Royer, and S. Das. RCF 3561 Ad Hoc On-demand Distance Vector Protocol (AODV). Network Working group, July 2003.

[5] OPNET Manual “www.OPNET/14.5A/doc/modeler10

[6] T. Clauser, ed.and P.Jacquet, ed. Optimized Link State Routing Protocol

[7] (OLSR). Network Working group, October 2003.

[8] V. Park and S. Corson. Temporally Ordered Routing Algorithm (TORA),

[9] IETF MANET working group, October 1999.

[10] Narendra Mohan Mittall and Savita Choudhary. Comparative Study of Simulators for Vehicular Ad-hocNetworks (VANETs), IJETAE Volume 4, Issue 4, April 2014.

[11] Saad Talib Hasson And Sura Jasim. Simulation Study to Observe the Effects of 
[12] Increasing Each of The Network Size and the Network Area Size on MANET's Routing Protocols. computerscijournal ISSN: $0974-6471$ April 2014, Vol. 7, No. (1): Pgs. 67-73.

[13] Jean-Daniel Medjo Me Biomo, Thomas Kunz, Marc St-Hilaire and Yifeng Zhou. Unmanned Aerial ad Hoc Networks: Simulation-Based Evaluation of Entity Mobility Models' Impact on Routing Performance. Aerospace 2015, 2, 392-422; doi:10.3390/aerospace2030392.

[14] Mustafa Almahdi Algaet, Zul Azri Bin Muhamad Noh, Abd Samad Bin Hasan Basari, Abdul Samad Shibghatullah, Ali Ahmad Milad and Aouache Mustapha. A Review of Service Quality in Integrated Networking System at the Hospital Scenarios. JTECE ISSN: 2180 - 1843 Vol. 7 No. 2 July - December 2015 . MohdRazeef and MohsinNazir, "Reliability of Software Development using Open Source Technology", in IJARCS, vol2, No. 5, pp. 479-485, Sept-Oct 2011 